\title{
Reanalyzing Inferred High Energy lonic Charge States for Solar Energetic Particle Events from 2005- 2016 with ACE and STEREO
}

\author{
A. W. Labrador ${ }^{1, *}$, L. S. Sollitt ${ }^{2}$, C. M. S. Cohen ${ }^{1}$, A. C. Cummings ${ }^{1}$, R. A. Leske ${ }^{1}$, \\ G. M. Mason ${ }^{3}$, R. A. Mewaldt ${ }^{1}$, E. C. Stone ${ }^{1}$, T. T. von Rosenvinge ${ }^{4}$, and \\ M. E. Wiedenbeck ${ }^{5}$ \\ ${ }^{1}$ California Institute of Technology, Pasadena, CA 91125 USA \\ ${ }^{2}$ Department of Physics, The Citadel, Charleston, SC 29409 USA \\ ${ }^{3}$ JHU/Applied Physics Lab, Laurel, MD 20723 USA \\ ${ }^{4}$ NASA/Goddard Space Flight Center, Greenbelt, MD 20771 USA \\ ${ }^{5}$ Jet Propulsion Laboratory, Pasadena, CA 91109 USA
}

E-mail: labrador@srl.caltech.edu

We have estimated mean high-energy ionic charge states of solar energetic particles (SEPs) using the Sollitt et al. (2008) method. In our analysis, the method applies to abundant elements (e.g. $\mathrm{N}, \mathrm{O}, \mathrm{Ne}, \mathrm{Mg}, \mathrm{Si}$, and $\mathrm{Fe}$ ) in SEP events at the energy ranges covered by the STEREO/LET instrument (e.g. 2.7-70 MeV/nuc for Fe) and the ACE/SIS instrument (e.g. 11$168 \mathrm{MeV} /$ nuc for Fe). The method starts by fitting SEP time-intensity profiles during the decay phase of a given, large SEP event in order to obtain energy-dependent decay times. The energy dependence of intensity decay times for a given element in an SEP event is combined with its assumed average atomic mass and an assumed average charge state of 5.9 for carbon as a reference element. The mean charge state for each element is then estimated from the relationship between the energy dependence of its decay times to that for carbon and all other elements in the event. The method has yielded charge states for 8 SEP events using ACE data, 5 SEP events with STEREO A data, and 4 SEP events with STEREO B data through 2015. Calculations for events before 2005 by Sollitt et al. using ACE data also incorporated helium time intensity profile fits, with an assumed charge state of 2, but later calculations dropped helium as a reference element for simplicity, as the calculations were first generalized to later events and to STEREO data. In this paper, we restore helium in order to refine the calculations and compare with the earlier results. In particular, we will focus on the 8 March 2012 event with ACE/SIS data and the 28 September 2012 event with STEREO/LET Ahead data. Additionally, other charge state measurements using a geomagnetic rigidity cutoff technique yielded correlations between charge states and $\mathrm{Fe} / \mathrm{O}$ ratio as well as to observed source solar longitudes. We will use the inferred charge state reanalysis to investigate whether these correlations are also present in ACE and STEREO data.

35th International Cosmic Ray Conference - ICRC2017

10-20 July, 2017

Bexco, Busan, Korea

Presenter 


\section{Introduction}

Solar energetic particles may be ionized in high temperature plasmas prior to acceleration, or through acceleration itself, or during propagation through relatively dense coronal or interplanetary material close to the sun [1-4]. The mean ionic charge states of solar energetic particle events generated by flares or coronal mass ejections may reflect the temperatures of the source plasmas, or they may indicate that the SEPs were accelerated out of source material ionized in prior SEP events.

The Mass Spectrometer Telescope aboard the Solar Anomalous and Magnetospheric Particle Explorer (SAMPEX/MAST), in polar Earth orbit, used a geomagnetic rigidity cutoff technique to measure mean ionic charge states, and SAMPEX/MAST measurements showed correlation between mean iron charge state $(\mathrm{Q}(\mathrm{Fe}))$ and the iron to oxygen ratio $(\mathrm{Fe} / \mathrm{O})[5,6]$. Similar correlation between $\mathrm{Q}(\mathrm{Fe})$ and $\mathrm{Fe} / \mathrm{O}$ ratio was observed at lower energies by the Solar Energetic Particle Ionic Charge Analyzer (SEPICA) using electrostatic deflection aboard the Advanced Composition Explorer (ACE) [7]. If the cause of such a correlation were to bias in favor of mass/charge, we would expect to see an anticorrelation instead, but acceleration from a previously ionized seed population could result in such correlation. Additionally, high $\mathrm{Q}(\mathrm{Fe})$ measurements have been observed to be more prevalent in SEP events in which the spacecraft are magnetically well connected to the solar source event.

In this paper, we report on ongoing analysis that infers mean ionic charge states through measurements of the energy-dependent time decay profiles of elements during certain SEP events. For this analysis, we use data from both the ACE spacecraft as well as the two STEREO spacecraft, for measurements up to several hundred $\mathrm{MeV} /$ nuc. In principle, observations by multiple spacecraft of a single SEP event will also yield longitude dependent measurements of mean ionic charge states. We demonstrate that these measurements are consistent with the charge to abundance ratio correlation observed by the other spacecraft as well as the correlation with the relative longitude of the solar source event.

\section{Inferred Ionic Charge States Measurement Technique}

We use the technique for inferring ionic charge states described by Sollitt et al. [8] with ACE/SIS data, and we have extended this method to use STEREO data [9]. The technique models SEP events as filling a magnetic containment volume in the interplanetary medium. After the injection of SEPs into the containment volume is complete, particles diffuse out of the containment volume exponentially vs. time. The escape time decay constant will be energy dependent as well, with higher energy particles escaping faster. Escape time decay constants are measured in intensity vs. time data, 
during the decay phase of a given SEP event. The time decay profiles must fit an exponential decay, or the method will not yield results, e.g. the decay phase of an SEP event should not be interrupted by passage of a strong shock front.

In the model, the time decay constant has the form

$$
\frac{1}{\tau_{X}}=\frac{1}{\tau_{y}}+W\left(\alpha_{x} E\right)^{\gamma}
$$

where $\tau_{\mathrm{X}}$ is the time decay constant as measured for a given element $\mathrm{X}$, measured during the decay phase of the SEP event, $\tau_{\mathrm{y}}$ is a low-energy decay time due to convection and adiabatic cooling, $\gamma$ is a constant arising from mean free path rigidity-dependence, and $\mathrm{W}$ is a normalization factor. The parameter $\alpha_{X}$ has the form

$$
\alpha_{x}=\left[\frac{Q_{c a l}}{A_{c a l}} \frac{A_{X}}{Q_{X}}\right]^{\frac{2 \gamma-1}{\gamma}}
$$

where $\mathrm{Q}$ and $\mathrm{A}$ are mean ionic charge states and mass number for elements $\mathrm{X}$ and some calibration element (cal), where carbon (C) was used as a calibration element with an assumed charge state of 5.9 by Sollitt et al. as well as our previous analysis [8-10]. The original Sollitt et al. analysis was specific to ACE/SIS data but also low energy He data from ACE/ULEIS to improve the fit for $\tau_{y}$, but we temporarily removed low energy $\mathrm{He}$ when we generalized the method to use data not only from ACE/SIS but also from STEREO/LET. We modified this analysis to use He data from either ACE/SIS or STEREO/LET instead as the calibration element, with an assumed charge state of 2 . We retained the use of $\mathrm{C}$ as a secondary calibration element with $\alpha_{\mathrm{C}}$ calculated from Equation 2, with a charge state of $\mathrm{Q}_{\mathrm{C}}=5.9$ as before, using $\mathrm{A}_{\mathrm{C}}=12, \mathrm{Q}_{\mathrm{He}}=2$, and $\mathrm{A}_{\mathrm{He}}=4$. Assuming these values in Equation 2 means that, although in principle there exists a parameter $\alpha_{C}$ that is variable during the fit of the model to the data, it depends solely on $\gamma$, so that $\alpha_{C}$ is not a free parameter for the purpose of the model fit. It is, therefore, not included as a separate parameter, and the $\mathrm{C}$ data with an assumed charge state thus provide calibration data in addition to He data. The constant $\gamma$ applies to all elements in a given SEP event, and it is fit by the algorithm as a variable parameter as described below.

We measure time decay constants during a given SEP event for a variety of elements ( $\mathrm{He}, \mathrm{C}, \mathrm{N}, \mathrm{O}, \mathrm{Ne}, \mathrm{Mg}, \mathrm{Si}$, and $\mathrm{Fe}$, when available) over available energy ranges from ACE/SIS element intensity vs. time data, STEREO/LET data, or both. Fits are applied to the exponential decay phase of a given SEP event, and if smooth exponentially decaying time periods are not available during the SEP event (e.g. passage of shocks distorting smoothly decaying profiles), the method is inapplicable for that event. Sollitt et al. [8] used maximum likelihood fits, but we have found that least-squares fits are consistent with maximum likelihood fits for sufficiently large, high-intensity SEP events. We select such events preferentially for this analysis. 
With measured time decays, $\tau_{\mathrm{X}}$, for several elements and a range of energies, the data are then fit to Equation (1). The downhill simplex algorithm ("amoeba") [11] varies all of the $\alpha_{x}$ 's, simultaneously, in order to find the best fit to Equation (1). Again, for this analysis, we use a least-squares fit, although a maximum likelihood fit may also be applied. Note that, with $\mathrm{He}$ and $\mathrm{C}$ used as calibration elements with assumed charges and masses, we need to find $\alpha_{x}$ 's for only $\mathrm{N}, \mathrm{O}, \mathrm{Ne}, \mathrm{Mg}, \mathrm{Si}$, and $\mathrm{Fe}$, the most abundant such elements available in ACE/SIS and STEREO/LET data for large SEP events. Thus, we have $6 \alpha_{x}$ 's plus $\mathrm{W}, \gamma, \tau_{\mathrm{y}}$, for a total of 9 parameters to fit to the measured time decay constants. The final, best-fit values for $\gamma$ and the $\alpha_{x}$ 's yield charge states $Q_{x}$ from Equation 1, and constant contours of maximum likelihood or $\Delta \chi^{2}$ yield charge state uncertainties, $\sigma_{\mathrm{Q}}$.

It is worth noting that fitting a 9 parameter model to a few dozen data points with the downhill simplex algorithm is sometimes unpredictable, and getting the algorithm to converge to a physical solution is something of an art. For example, the selection of start and stop times for the time decay profile measurements can yield different final results or no results. Additionally, the selection of 10 points in 9-dimensional parameter-space to form the initial simplex yield a far greater number of possibilities for starting the downhill simplex algorithm. With the chisquare manifold in that space likely being complex, with many local minima, depending on the data set to be fit, a range of possible, different starting simplexes can converge to a variety of local minima in chisquare, resulting in different parameter fits or even a failure to converge to a solution. In practice, the downhill simplex is run two or more times with different starting simplexes, with the expectation that convergence with different initial simplexes to the same or similar set of fit parameters means that the repeatable solution is robust and acceptable. Whether a failure to converge to a solution depends on the time selection or on the initialization of the downhill simplex algorithm is practically impossible to determine.

Additionally, not all SEP events may yield results with this technique. If a given SEP event is not sufficiently large, time decay profiles may not be measured with useful statistical accuracy, and the method may not converge to a solution or may yield nonphysical results. Additionally, we find that not all SEP events exhibit smoothly varying energy dependence in time decay constants as given in Equation 1, with more energetic particles expected to escape with smaller decay constants than those of less energetic particles. In such cases, parameters may not be established to allow the model to fit the data. Or the energy dependence may be weak, so that the resulting chisquares are broad and shallow in parameter-space, resulting in the downhill simplex algorithm not converging within its programmed tolerances [11]. Additionally, such weak energy dependence will also result in large uncertainties in final charge states.

\section{Results}

For this work, we have reanalyzed the 8 March 2012 SEP event in ACE/SIS data and the 28 Sept 2012 event in STEREO/LET Ahead data. We previously reported results of 
these events with the analysis using only $\mathrm{C}$ as the calibration element, with an assumed $\mathrm{Q}(\mathrm{C})=5.9$ [10]. The 8 March 2012 event was the most intense of the St. Patrick's Day series of events that caused multiple reboots and data outages on NASA spacecraft [12].

The results of the fit to Equation 1 are shown in Figure 1 ( $a$ and $b$ ). This analysis uses $\mathrm{He}(\mathrm{Q}(\mathrm{He})=2)$ and $\mathrm{C}(\mathrm{Q}(\mathrm{C})=5.9)$ to calibrate the fits to the parameters $\alpha_{\mathrm{X}}$, in Equation 2 . Initial attempts to use only $\mathrm{He}$ as the calibration element resulted in nonphysical charge states, e.g. ionization states greater than nuclear charge. Therefore, $\mathrm{C}$ was retained as an additional calibration element, as a lever arm to help limit higher $\mathrm{Z}$ charge states.

The resulting ionic charge states are shown in Figure 2 ( $a$ and $b$ ), comparing the previous ( $\mathrm{C}$ only calibration) charge states with charge states in the current analysis ( $\mathrm{He}$ and C for calibration), for both the 8 March 2012 and 28 September 2012 SEP events. The fit uncertainties are shown, and the results of the two analyses are consistent with one another.

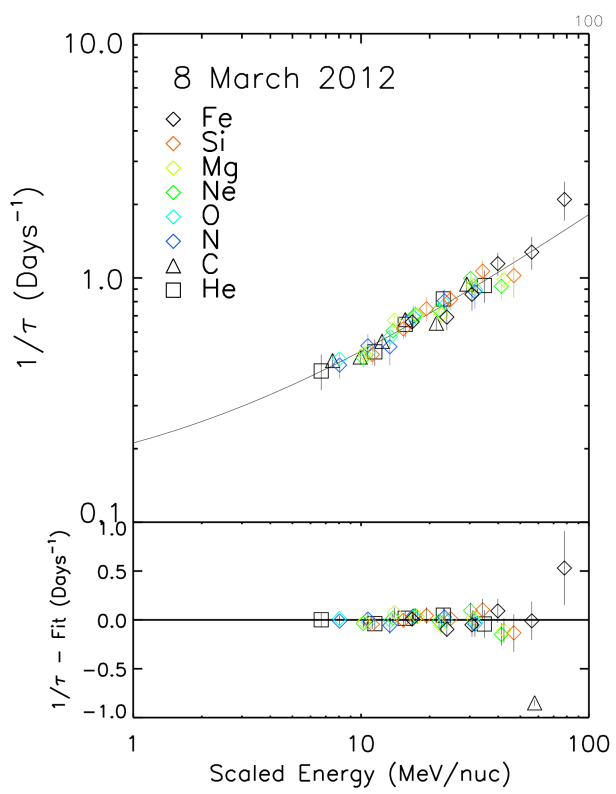

(a)

Figure 1 (a, left, and $b)$ : Fits of decay times vs. energy for elements in the 8 March 2012 and 28 September 2012 SEP events, with residuals in the lower panel of each plot. The 8 March 2012 event shows ACE/SIS data, and the 28 September 2012 events shows STEREO/LET A data. The scaled energies of the data points are the measured energies multiplied by the corresponding parameters, $\alpha_{X}$

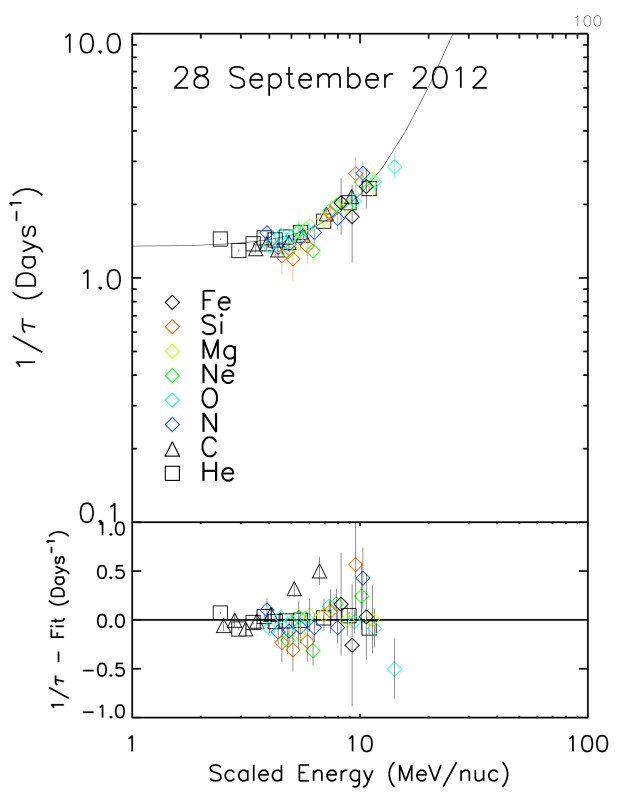

(b) 


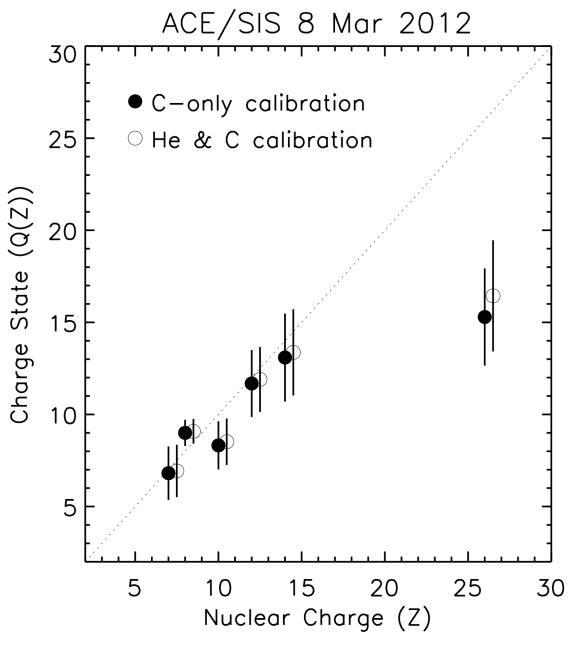

(b)

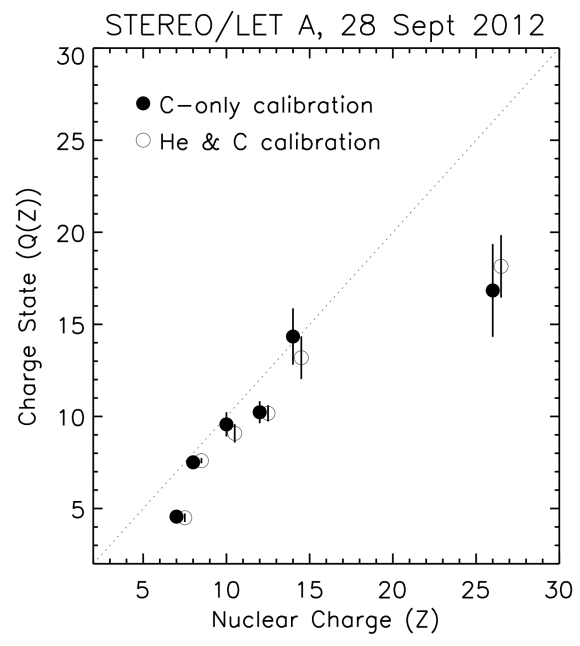

Figure 2 ( $\mathrm{a}$ and $\mathrm{b}$ ): Comparisons of inferred charge states with only $\mathrm{C}$ as the calibration element (filled circles) to inferred charge states with $\mathrm{C}$ and $\mathrm{He}$ as the calibration elements (open circles, offset). The events are from 8 March 2012 and 28 September 2012.
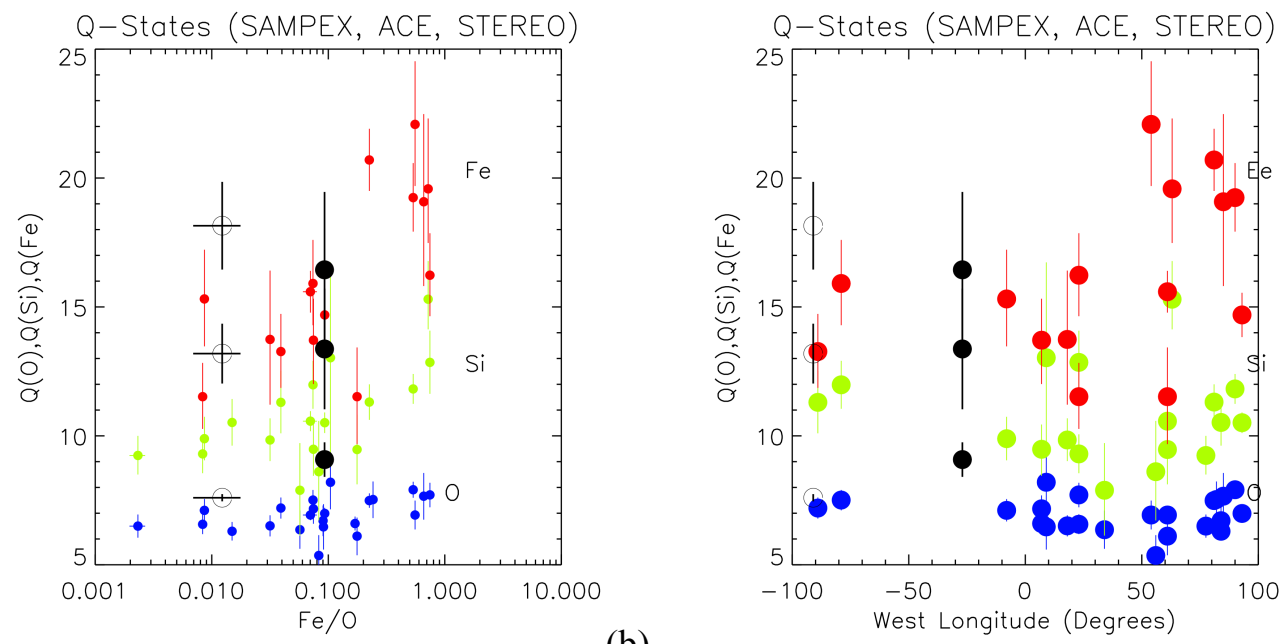

(a)

(b)

Figure 3: (a, left) Ionic charge states for $\mathrm{O}, \mathrm{Si}$, and $\mathrm{Fe}$ vs. Fe/O ratio, and (b) ionic charge states vs. solar source longitude of the each SEP event's source flare relative to the measuring spacecraft [13]. The colored points are SAMPEX/MAST results $[5,6]$, the solid black circles are results for the 8 March 2012 event from this analysis ( $\mathrm{H}$ and $\mathrm{C}$ as calibration elements), and the open circles are results for the 28 September 2012 event also from this analysis.

Figure 3 shows comparisons between inferred ionic charge state results for the 8 March 2012 and 28 September 2012 SEP events, from this analysis with the He and C as calibration elements. In the charge states vs. Fe/O ratio correlation plot, the newer 
measurements are roughly consistent with the previously observed correlation, though the 28 September 2012 charge states are at the higher end of nearby measurements. The newer measurements are also consistent with previous measurements showing that high Fe charge states, $\mathrm{Q}(\mathrm{Fe})$, are associated with western relative longitudes, indicating source flare locations that are magnetically well-connected to the measuring spacecraft. In both figures, however, the $\mathrm{Q}(\mathrm{Fe})$ values are the highest of the "low $\mathrm{Q}(\mathrm{Fe})$ " values, though the uncertainty is large enough to overlap clearly with the several of the other low $\mathrm{Q}(\mathrm{Fe})$ measurements.

\section{Conclusions}

The new analysis adding $\mathrm{He}$ as a calibration element in the inferred charge state measurement technique yields results that are consistent with previous measurements using only $\mathrm{C}$ as a calibration element, although $\mathrm{C}$ had to be retained to help prevent non-physical results for the two events in this analysis. The measurements remain consistent with the body of earlier measurements using different measurement techniques (e.g. geomagnetic rigidity cutoff) that show charge state vs $\mathrm{Fe} / \mathrm{O}$ ratio correlation and measurements that show high charge states associated with magnetically well-connected source event locations.

\section{Acknowledgments}

This work was supported by NASA grant NNX13A66G and NASA contract NA5503131.

\section{References}

[1] G.M. Mason et al., Solar Cycle Abundance Variations in Corotating Interaction Regions: Evidence for a Suprathermal Ion Seed Population, The Astrophysical Journal Letters 748 L31 (2012).

[2] E. Moebius et al., Strong Energy Dependence of Ionic Charge States in Impulsive Solar Events, Proc. 28th International Cosmic Ray Conf., 6, 3273 (2003).

[3] B. Klecker et al., Ionic Charge States of Solar Energetic Particles: A Clue to the Source, Space Science Reviews 130273 (2007).

[4] R.A. Mewaldt et al., Energy Spectra, Composition, and Other Properties of Ground-Level Events During Solar Cycle 23, Space Science Reviews 17197 (2012).

[5] R.A.Leske et al., Measurements of the Ionic Charge States of Solar Energetic Particles Using the Geomagnetic Field, The Astrophysical Journal Letters. 452, L149 (1995).

[6] A.W. Labrador et al., High Energy Ionic Charge State Composition in the October/November 2003 and January 20, 2005 SEP Events, Proc. 29th International Cosmic Ray Conf., Pune, 1 99 (2005). 
[7] E. Moebius et al., Survey of Ionic Charge States of Solar Energetic Particle Events During the First Year of ACE, AIP Conf. Proceedings 528131 (2000).

[8] L.S. Sollitt et al., A novel technique to infer ionic charge states of solar energetic particles, The Astrophysical Journal 679910 (2008).

[9] A.W. Labrador et al., Inferred Ionic Charge States for SEPs with ACE and STEREO, Proc. 33rd International Cosmic Ray Conference, Rio de Janeiro (2013).

[10] A.W. Labrador et al., Inferred Ionic Charge States for Solar Energetic Particle Events from 2012-2015 with ACE and STEREO, Proc. 34 International Cosmic Ray Conference, The Hague (2015).

[11] J.A. Nelder and R. Mead, A simplex method for function minimization, Computer Journal 7 (1965) 308313. doi:10.1093/comjn1/7.4.308.

[12] M. Guhathakurta, EOS 94, 165 (2013).

[13] I.G. Richardson et al., > 25 MeV Proton Events Observed by the High Energy Telescopes on the STEREO A and B Spacecraft and/or at the Earth During the First Seven Years of the STEREO Mission, Solar Physics, 289:30593107 DOI 10.1007/s11207-014-0524-8, 2014. 